\title{
Optical characterization of surfaces by robust reflectance determination based on air-gap interference
}

\author{
J.C. Martinez-Antón*, J.A. Quiroga, R. González-Moreno, E. Bernabeu \\ Departamento de Óptica, Facultad de Ciencias Físicas, Universidad Complutense Madrid, Ciudad universitaria s/n, 28040 Madrid, Spain
}

Available online 20 July 2004

\begin{abstract}
In this work we present an optical tool for characterizing the reflectance and polarimetric properties of surfaces. It uses only the image of the interference fringe pattern produced in a thin air-gap between the surface of interest and a glass surface acting as a reference. From only the contrast of the fringe pattern captured with a CCD we may obtain the reflectance of the surface, no need of measuring a reference beam. By taking two images with polarized light, we may get then the polarized reflectance $R_{\mathrm{p}}$ and $R_{\mathrm{s}}$, but also the ellipsometric magnitude $\Delta$, simply as a phase shift between fringes in $\mathrm{p}$ and s polarization. A sample of silicon with a thin layer of thermally grown silica is used to test the method.
\end{abstract}

(C) 2004 Elsevier B.V. All rights reserved.

Keywords: Photo-interferometric envelope analysis; Newton rings; Optical characterization; Spectrophotometry

\section{Introduction}

The optical interference produced in deposited thin films is commonly used to obtain the optical thin film parameters (thickness, refractive index, absorption, etc.) by means of the extremes and the envelopes of reflectance or transmittance spectra [1]. The observation of interference is limited to thickness above $\sim 0.2 \mu \mathrm{m}$ for the visible range. Spectrophotometry is also used for bare substrates but is often considered as being not very sensitive for very thin films, specially in transmittance. In reflectance, the main limitation is that accurate and precise measurements are not easy to perform. In certain cases reflectance measurements are preferable or even unavoidable (for non-transparent samples).

\footnotetext{
* Corresponding author. Tel.: +34 91394 4629; fax: + 34913944683 .

E-mail address: jcmartin@fis.ucm.es (J.C. Martinez-Antón).
}

Here we present a non-conventional approach to measure the reflection properties of a surface and therefore to obtain its optical characterization with some advantages. We base our method in the use of the interference produced in a film, but now the film is the tool not the objective. A thin air-gap between a reference glass and the surface to explore produces interference in reflected light which can be analyzed by using some methods already known. We will show how to obtain the reflectance of the surface of interest in a simple and robust way by using this scheme.

Typically, to measure the reflectance of a surface we need two independent inputs, one measuring the reference or the incident beam and the other beam probing the sample of interest. Stability of the light source and critical alignment are among the problems we may encounter to have a reliable measurement. In the proposed technique of imaging interference patterns, we need only one measurement and it is virtually free of the mentioned problems, because it uses 
only the contrast of the fringes. A similar methodology has been presented recently [2] but using spectral reflection scans from a fixed area of the surface. In the approach introduced here, the illuminating wavelength is fixed and we get images of the interference modulation at the surface produced by the non-uniform thickness of a trapped air-gap between the surface of interest and a glass surface. In both cases the parameter versus we get interference is $\lambda / d$ (Fig. 1), but now, we do not have the potential problem of spectral features which may be difficult to handle if very pronounced.

Consider the experimental scheme of Fig. 1. The first surface will act as a reference standard, but in fact, we do not need to measure the incident beam as in conventional spectrophotometry, since the reflected spectra contains both the information of the surface and of the reference glass. The envelopes of fringes (maximums and minimums) will permit us to discriminate between both surfaces. Therefore, from two spectra (at $\mathrm{p}$ and $\mathrm{s}$ polarization) we will obtain at least, the reflectance of the surface at $\mathrm{p}$ and $\mathrm{s}$ polarization. Finally, by comparing the locations of the envelope extremes, we obtain the ellipsometric phase $\Delta$ produced at the studied surface.

Some experimental results are given based on the imaging of interference fringes in an arbitrary airgap. We measured a silicon wafer with a thermally grown oxide layer of controlled thickness $(d=0.1625 \mu \mathrm{m})$.

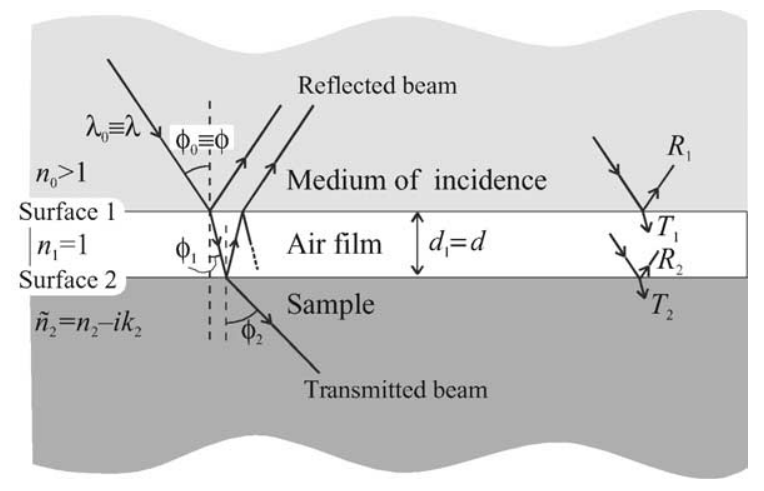

Fig. 1. Experimental scheme and parameters. An air-gap is sandwiched between the medium of incidence (e.g. a glass prism) and the surface to measure. This produces an interference modulation of the reflected beam. In the figure the sample appears as an absorbing substrate but in fact, it can be any optically flat stratified surface.
The main advantage of the method is its robustness. Compared to conventional spectrophotometry, there is no need of a careful alignment or a very stable light source.

\section{Method}

Consider the experimental scheme and parameters of Fig. 1. We may write the reflectance as [1-3]:

$R=\frac{R_{1}+R_{2}+2 \sqrt{R_{1} R_{2}} \cos \left(2 \beta+\varphi_{1}-\varphi_{2}\right)}{1+R_{1} R_{2}+2 \sqrt{R_{1} R_{2}} \cos \left(2 \beta-\varphi_{1}-\varphi_{2}\right)}$,

for both $\mathrm{s}$ and $\mathrm{p}$ polarization, being $R_{\mathrm{i}}=r_{\mathrm{i}} r_{\mathrm{i}}^{*}$ the reflectance at each interface and

$r_{\mathrm{i}}=\sqrt{R_{\mathrm{i}}} \exp \left(j \varphi_{\mathrm{i}}\right)$,

the reflection coefficients, where $\varphi_{\mathrm{i}}$ are the respective reflection phase shifts. The phase term $\beta$ of the air-gap $\left(n_{1}=1\right)$ is:

$\beta=\frac{2 \pi d}{\lambda} \sqrt{1-n_{0}^{2}(\lambda) \sin ^{2}(\phi)}$.

As explained in [1], we may write the envelopes for the reflectance $R$ as:

$E_{R \pm}=\left[\frac{\sqrt{R_{1}} \pm \sqrt{R_{2}}}{1 \pm \sqrt{R_{1} R_{2}}}\right]^{2}$

The double sign holds for the envelope of the maximums (+) or for the envelope of the minimums (-). This expression is valid under the assumption that the reflection phase at the first surface $\varphi_{1}$ is $m \pi(m=0,1$, 2 ), which is valid for a neat glass surface, except for the close surroundings of Brewster angle at $p$ polarization if the surface is not mathematically perfect (or Fresnel surface).

Consider now imaging the set-up of Fig. 1 with an airgap film of non-uniform in thickness $d$. A reflection fringe pattern $I(d)$ can be obtained for $\mathrm{p}$ and s polarization (see Fig. 2). The reference spectrum $I_{0}$ valid to compute the reflectance of the sample is unknown. However, from expression (4) we may write that:

$E_{I \pm}=I_{0}\left[\frac{\sqrt{R_{1}} \pm \sqrt{R_{2}}}{1 \pm \sqrt{R_{1} R_{2}}}\right]^{2}$.

where $E_{I \pm}$ are the envelopes for the reflection signal $I(d)$. We may eliminate the dependence from the 
unknown reference $I_{0}$ in (5) by combining the envelopes of maximums and minimums to achieve the following expression:

$\frac{\sqrt{E_{I+}}-\sqrt{E_{I-}}}{\sqrt{E_{I+}}+\sqrt{E_{I-}}} \equiv V_{\mathrm{R}}=\frac{R_{1}}{R_{2}} \frac{1-R_{2}^{2}}{1-R_{1}^{2}}$

where $V_{\mathrm{R}}$ is a contrast function defined in terms of the envelopes of the reflection signal $I . R_{\mathrm{i}}$ are the reflectance of the interfaces delimiting the air film (see Fig. 1). From the measure of $V_{\mathrm{R}}$ and the knowledge of $R_{1}$ we finally obtain the reflectance of the sample $R_{2}$ as desired. In the previous expression it was assumed that $R_{1}<R_{2}$. For the opposite case $\left(R_{2}>R_{1}\right)$ we must invert the right term of Eq. (6). In practical situations, the proper choice is known from previous qualitative information or by discarding non-sense solutions. From two images at a fixed wavelength and at $\mathrm{p}$ and $\mathrm{s}$ polarization respectively, we can get $R_{\mathrm{p}}$ and $R_{\mathrm{s}}$ for our sample (and therefore the ellipsometric $\tan \varphi$ too) from Eq. (6).

Let estimate now $\Delta$ from the location of fringes. Strictly, we must use the envelope-extremes, which are the contacting points of the envelopes with the reflection profile and not merely maximums and minimums. Anyway, in a typical experimental setup the irradiance is kept nearly constant over the surface, therefore the envelope extremes coincide with the maximums and minimums. From the distance between corresponding envelope extremes relative to a period we may obtain the term $\Delta=\varphi_{2 \mathrm{p}}-\varphi_{2 \mathrm{~s}}$.

The situation of the envelope-extremes is governed by the expression (see [1])

$2 \beta-\varphi_{2}=N \pi, \quad N=0, \pm 1, \pm 2, \ldots$,

or in a more explicit form (expanding $\beta$ )

$N=\frac{4 d}{\lambda} \cos \left(\phi_{1}\right)-\frac{\varphi_{2}}{\pi}$,

where remember we assumed that the reflection phase at the first surface $\varphi_{1}$ is $m \pi(m=0,1,2)$. In the last expression only the reflection phase term $\varphi_{2}$ is dependent on polarization and the other right term is simply geometrical, and then constant for two images taken at p or s polarization. The parameter $\Delta=\varphi_{2 \mathrm{p}}-\varphi_{2 \mathrm{~s}}$ can be obtained by comparing both images and eliminating the geometrical term. Two general methods have been used to obtain $\Delta:$ : (1) a calculation of the phase all over the sample by conventional image processing techniques of fringe patterns [5] and then subtracting both maps of phase (p and s). (2) The second method uses intensity profiles over a line in the images and compares the location of minimums (better defined

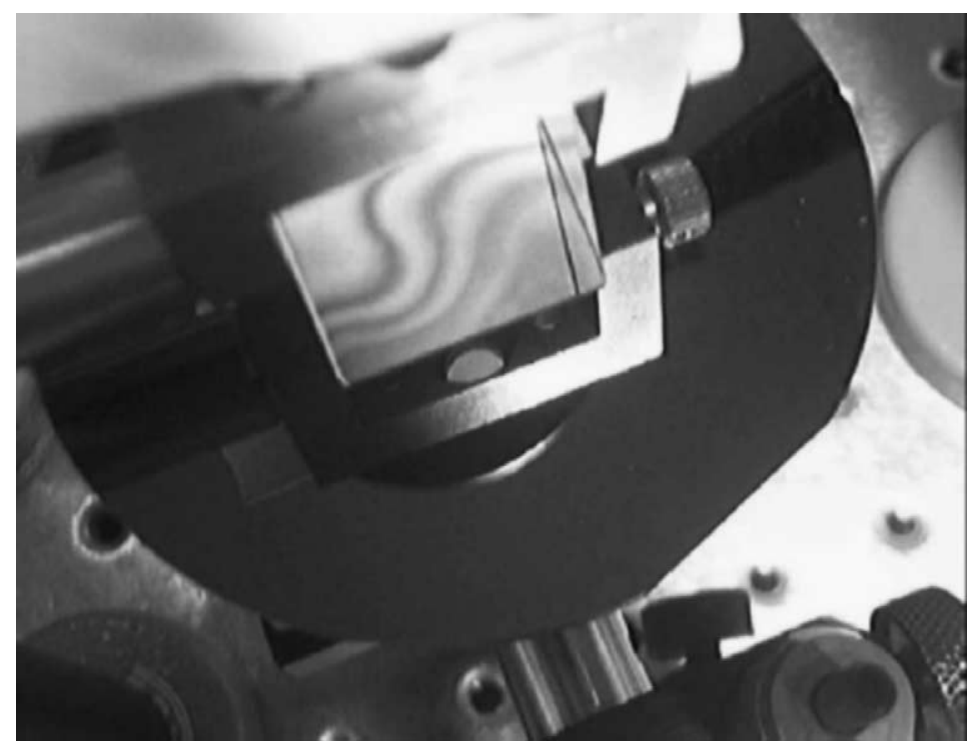

Fig. 2. Picture of the sample set-up. A prism of $\mathrm{SiO}_{2}$ is situated on the surface to characterize. In this case a silicon wafer thermally oxidized. In the air-gap between them we may appreciate interference fringes due to a non-uniform air-gap thickness. 
than the maximums in our experimental situation). Both methods lead to similar results, although in the first method, as the fringes are not harmonic we have to average carefully.

\section{Experimental results}

We use the set-up pictured in Fig. 2 to measure a calibration sample $\left(\mathrm{SiO}_{2}\right.$ on silicon with a thickness of $0.1625 \mu \mathrm{m}$ ) and test the method proposed. To obtain the images we use CCD camera with a telecentric objective in order to keep the angle of incidence constant over the field of view. The source is a
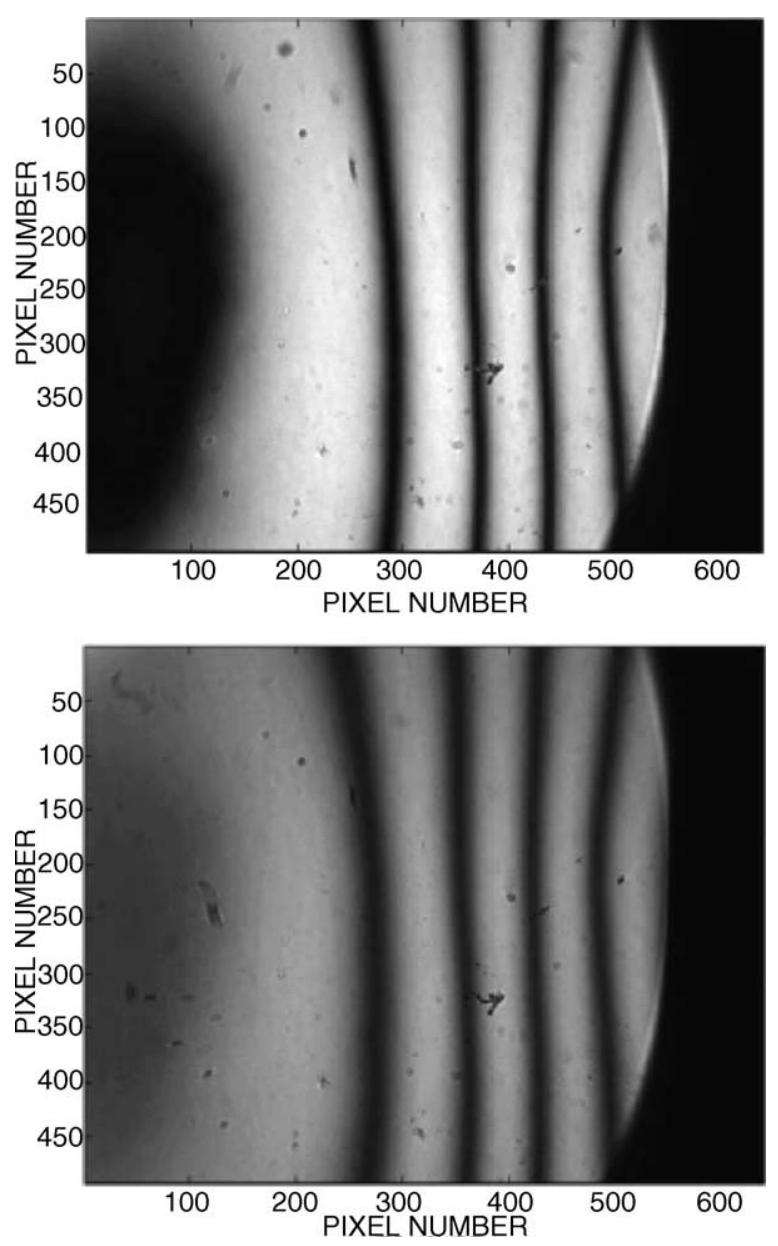

Fig. 3. Captured images for later processing (above in $s$ polarization, below in $\mathrm{p}$ polarization). collimated white light beam. The system limits the angular bandwidth to $\sim 0.1^{\circ}$. To have a quasi-monochromatic light we used interferential filters centered at 610,589 and $546 \mathrm{~nm}$ with a nominal bandwidth of $\Delta \lambda=10 \mathrm{~nm}$. The angle of incidence $\Psi_{1}$ was set to $73.6 \pm 0.3^{\circ}$ for $\lambda=589 \mathrm{~nm}$. The angle of incidence varies for other $\lambda$ due to the dispersion of the prism.

We obtained the images of Fig. 3. To estimate the contrast we used a well resolved area of the images and apply a histogram analysis (see [6]) to get the maximums and minimums of the fringes contained in the images or, a direct measurement using intensity profiles of the fringe pattern (see Fig. 4). The first method is sensitive to an irradiance not constant over the explored surface. We can not guarantee this condition so we preferred to compare the results of the second method. Finally, for the comparison between experimental and expected values we take into account the instrumental function for the computation of expected values (consider that the incident beam is not perfectly collimated $\left(\sim 0.1^{\circ}\right)$ and is not monochromatic $(\Delta \lambda=10 \mathrm{~nm}))$. This comparison is shown in Fig. 5.

From the estimation of fringe locations we get the following results for $\Delta$. We obtained experimentally that $\Delta=1.04 \pm 0.06 \mathrm{rad}$ for all of the wavelengths. Expected values are [4]: $\Delta(610 \mathrm{~nm})=1.16$, $\Delta(589 \mathrm{~nm})=1.17$ and $\Delta(546 \mathrm{~nm})=1.21$. There is an apparent systematic misfit that we interpret in

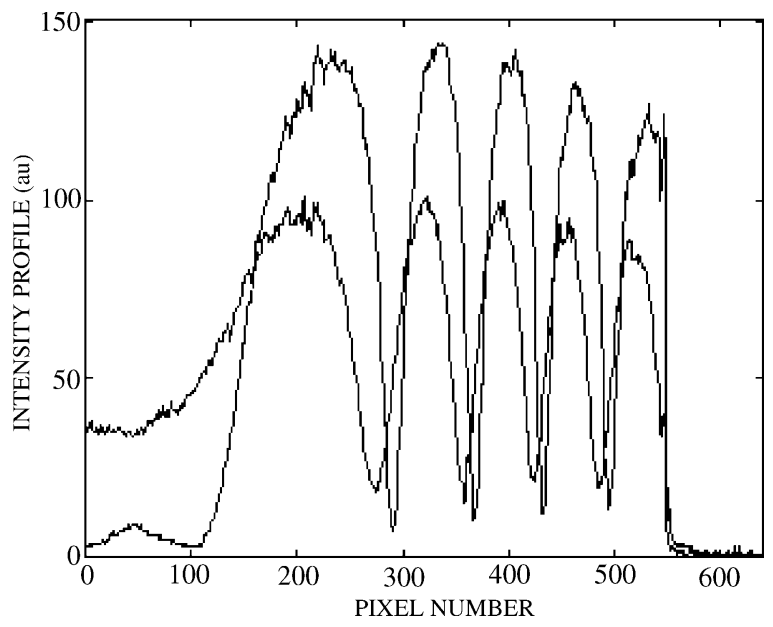

Fig. 4. Intensity profiles from the images of Fig. 3. After processing the location of minimums we obtain $D$ values (see text). 


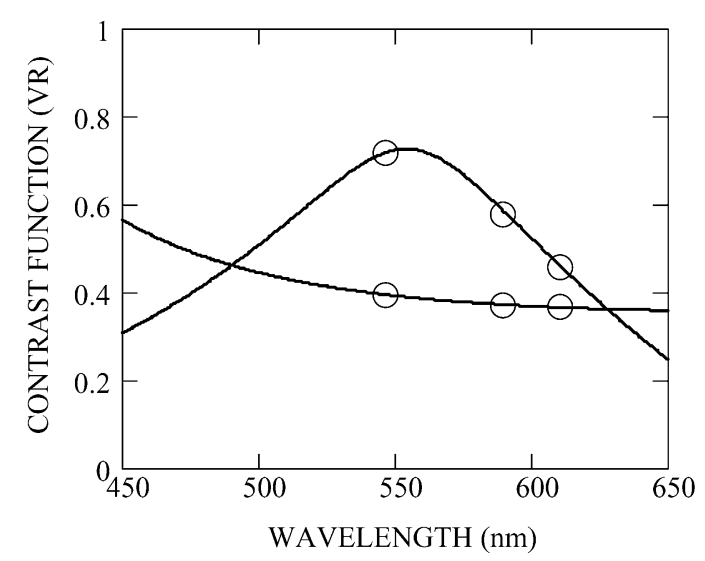

Fig. 5. Envelope contrast function $V_{\mathrm{R}}$ for s polarization (curve above) and p polarization (curve below). The experimental data is represented with circles and the expected values with a continuous line.

terms of the assumption of making $\varphi_{1}$ as $m \pi$. A very slight surface roughness in the glass of reference will contribute to the value of $\Delta$ in a significant way (e.g. $\sim 10^{\circ}$ ) which is not accounted for in our comparison an may explain this slight discrepancy.

\section{Conclusion}

We have introduced and tested a photo-interferometric method to measure reflectance and the relative reflection phase shifts. It is based on the imaging of interference in a thin air-gap between a glass and the surface of interest. From a minimum of two reflection fringe pattern images (one at $\mathrm{p}$ and other at s polarization) we get three magnitudes: $R_{\mathrm{p}}, R_{\mathrm{s}}$ and $\Delta$.

We do not need to know the irradiance of the incident beam to obtain the reflectance of the surface of interest. But, we still rely on the knowledge of the first surface or glass working as a reference or reflectance standard. However, contrary to conventional spectrophotometric techniques, we do not need to align elements or maintain a very stable light source. The reference information is already contained in the reflection profile which is analyzed by means of the interference envelopes. The expected resolution for $\Delta$ depends on the ability of positioning the envelope extremes, typically between $\sim 1 / 25$ and $1 / 500$ of the distance between extremes, depending mostly on the signal to noise ratio. This range of uncertainty leads to a resolution of $\Delta$ in the range of $0.4-8^{\circ}$.

In our experimental approach we have detected several aspects to take care. The method is sensitive to spectral bandwidth, the degree of collimation and the sampling resolution of fringes limited by the CCD. All of these instrumental factors can reduce the contrast of envelopes. A simple way to reduce its impact is using a very thin layer of air-gap (in our case around $1-2 \mu \mathrm{m})$.

\section{Acknowledgements}

This work has obtained partial financial support from the project DPI.2001-1238 (MCYT, Spain).

\section{References}

[1] J.C. Martínez-Antón, Determination of optical parameters in general film-substrate systems. A reformulation based on the concepts of envelope-extremes and local magnitudes, Appl. Opt. 39 (35) (2000) 4557-4568.

[2] J.C. Martinez-Anton, O. Esteban, Photo-interferometric spectroscopic ellipsometry, Thin Solid Films 455-456 (2004) 90-94.

[3] R.M.A. Azzam, N.M. Bashara, Ellipsometry and Polarized Light, North-Holland, Amsterdam, 1977, pp. 283-285.

[4] E.D. Palik, Handbook of Optical Constants of Solids, vol. 1, Academic Press, New York, 1985.

[5] M. Malacara, M. Servin, Z. Malacara, Interferogram Analysis for Optical Testing, Marcel Dekker, 1998.

[6] L.M. Sánchez-Brea, J.A. Quiroga, A. García-Botella, E. Bernabeu, Histogram-based method for contrast measurement, Appl. Opt. 39 (23) (2000) 4098-4106. 